\title{
Primary Fallopian Tube Carcinoma
}

\author{
Nicolae Bacalbașa1, Beatrice Lintoiu², Irina Bălescư3, Mihai Dimitriu
}

I"Carol Davila"University of Medicine and Pharmacy, Bucharest, Romania

2"Profesor Dr. Agripa Ionescu" Clinical Emergency Hospital, Bucharest, Romania

3"Ponderas"Hospital, Bucharest, Romania

4"St. Pantelimon" Clinical Emergency Hospital, Bucharest, Romania

\author{
Corresponding author: \\ Nicolae Bacalbașa, MD \\ Dimitrie Racoviță Street, no. 2 \\ Bucharest, Romania \\ E-mail: nicolae_bacalbasa@yahoo.ro
}

\section{ABSTRACT}

Primary fallopian tube carcinoma (PFTC) is a rare gynecological malignancy. Its preoperative diagnosis can be missed or delayed due to the lack of specific symptoms and signs. It is difficult to distinguish from serous epithelial ovarian cancer or primary peritoneal serous carcinoma during or even after operation because they have the same histopathological features. Standard guidelines regarding the optimal management are still not well defined. Key words: primary fallopian tube carcinoma, serous ovarian carcinoma, CA-125 levels

\section{INTRODUCTION}

Primary cancer of the fallopian tube is very rare, with an incidence of 0.14 to $1.8 \%$ of all gynecological cancers. An average of 20 to 30 new cases is reported each year. In 1888 it was first described by Orthmann.

The rarity of this cancer is due to the fallopian tube low oncogenic potential, in contrast to the vulnerability of the organ to infection. It is generally of poor prognosis.

The true incidence is underestimated. The diagnosis can be made wrongly as ovarian cancer during the initial surgery or even during the examination and pathology. Cancer of the fallopian tube occurs most often after the fourth decade of life, with an average age of 62 years (17-88 years). No case was reported in childhood (1-3).

\section{CLINICAL PRESENTATION}

Preoperative diagnosis is extremely difficult because PFTC is often asymptomatic and it is frequently misdiagnosed as ovarian tumor or pelvic inflammatory disease (4). Abdominal pain, vaginal bleeding, hydro-hematorrhea and palpation of a pelvic mass are the most common symptoms and signs. 
The consultation period for patients with cancer of the fallopian tube is shorter than that of patients with ovarian cancer, because of abdominal pain secondary to distention of the fallopian tubes (5). The Latzko triad of symptoms, including an intermittent, profuse, serosanguinous vaginal discharge, a colicky pain, often relieved by the discharge, and abdominal or pelvic mass, has been reported in $15 \%$ of patients with PFTC (6).

Although abdominal pain is one of the most frequently noted symptom, less than $50 \%$ of all patients with PFTC were found at an earlier stage (FIGO I/II) (7).

An early cervicovaginal cytological diagnosis may reveal some cases of silent PFTC because the malignant cells, which may exfoliate from the primary tumors, migrate through the fallopian tube and are deposited in the vaginal pouch or cervix canal. Some characteristic features of cervicovaginals mears might suggest the possibility of PFTC, including the clean background, which disappears when liquid-based cytology is used, the small number of malignant cells and the papillary grouping of overlapped neoplastic cells (8).

\section{IMAGING EVALUATION}

Imaging diagnosis of PFTC is important because it can help planning adequate initial surgery. Similarly to other gynaecologic tumours, the imaging approach includes ultrasound as the initial modality, followed by MRI and CT. CT is only used for staging purposes and not for pelvic mass characterization due to low softtissue contrast in comparison to MRI $(9,10)$.

Adominopelvic ultrasound often shows a retro or latero uterine heterogeneous mass, with different sizes (11). Anechoic or low-level echoes with papillary projections or intraluminal masses are an indication of PFTC. However, most of the echographic appearances of the fallopian tubeare nonspecific, mimicking other pelvic diseases such as tubo-ovarian abscess, ovarian tumor and ectopic pregnancy. The appearance of PFTC is usually based on the dominant component the solid tumor or the hydrosalpinx, which may be altered with serial imaging, reflecting the change in the amount of serous fluid within the tube. The former appears as a sausage-shaped adnexal mass and the latter appears as a fluid-filled tubular adnexa structure, containing nodular or papillary solid components, or a multilocular cystic mass with a cogwheel pattern (12). On Doppler ultrasound, a low-impedance flow within the solid components may be a clue. Variability of imaging characteristics in serial imaging may also point towards PFTC (9).
MRI is the modality of choice for evaluating an undetermined pelvic mass on ultrasound (13). Recently Ma et al. (10) have outlined the use of MRI features for differentiating PFTC from epithelial ovarian cancer (EOC). The characteristic appearance of PFTC is a relatively small, tubular-shaped (or sausage-shaped) mass, with homogenous signal, low signal intensity on T1 images high signal intensity on T2 images, mild to moderate enhancement, and hydrosalpinx or intrauterine fluid. The authors identified tubular shape, hydrosalpinx, and the presence of intra-uterine fluid as the most specific direct and indirect signs of PFTC. The combination of an adnexal mass with at least one of the former features yields a high diagnostic accuracy.

The normal fallopian tubes are usually not visualized on pelvic MRI. In the presence of intraperitoneal fluid, they may be seen as paired thin structures, extending from the ovaries to the uterine cornua, in the superior edge of the broad ligament. They extend for about 10$12 \mathrm{~cm}$ and are divided into four portions: the intramural/interstitial on the medial end, the isthmus, the ampulla and the infundibulum at the lateral fimbriated end (14).

The tubular nature of these paired organs is seen behind the sausage-shaped appearance when they are filled with solid tumour. This is one of the most specific signs seen in PFTC, particularly in the presence of contrast enhancement of the mass. Other pathologies such as a tubo-ovarian abscess can present with hydrosalpinx and solid component, thus differentiation from malignancy can be challenging.

Hydrosalpinx forms in PFTC both due to the fluid produced by the tumour and to the partial obstruction of the tubes. This leads to tubular distention and MRI can easily detect the presence of hydrosalpinx, which usually appears as a cystic, tubular shaped, convoluted mass, with well-defined walls. The dilated tubes contain incomplete folds, producing its convoluted appearance, either seen as the "waist sign", causing focal constriction of the tubular structure, or as the "beak sign", reflecting an acute angular contour, which would not be seen on a regular tubular or round structure (15). This is distinct from the "beak sign" described as an indicative feature of an intra-ovarian lesion. When a fallopian tube is extremely dilated by fluid, it may fold upon itself to produce the "cogwheel sign", which may be indistinguishable from an ovarian neoplasm. Occasionally, the "synechiae sign", consisting of fine strands running across the lumen, can also be seen indilated fallopian tubes $(15,16)$. The patency of the fallopian tube can lead to discharge of fluid into either the intra-uterine or the peritoneal cavities. However, 
ascites is not a specific sign of PFTC and is frequently present in ovarian cancer. Intra-uterine fluid, on the other hand, is specific of PFTC and it can occur in up to $30 \%$ of cases (10). So, PFTC can be suspected on MRI in the presence of features related to fallopian tube disease (9).

However, diagnosis is rarely made preoperatively. The concept of pelvic pain in the history of the disease, due to distension of the tubes, combined with latero uterine pelvic mass on imaging should suspect a tumor of the fallopian tube. Intraoperative diagnosis itself is not possible in $50 \%$ of cases due to tumor extension (17). And even when the tumor is resected, the diagnosis might be misinterpreted as ovarian cancer on pathological examination $(7,17)$.

\section{TUMOR MARKERS}

Many tumor markers have been reported to be valuable preoperatively to increase diagnostic accuracy and postoperatively to monitor the response after treatment or detect recurrence. CA-125 is the most commonly used as $80 \%$ of patients with PFTC have elevated pretreatment serum levels of CA-125 (16). Hefler et al (18) found that the sensitivity, specificity, positive predictive value and negative predictive value of serum CA-125 levels in the follow-up of patients with PFTC were $92 \%, 90 \%, 67 \%$, and $98 \%$, respectively. Moreover, the serum CA-125 level was found to correlate independently with disease-free survival and overall survival, adequately reflect patient response to chemotherapy and precedewith a median lead time of 3 months the clinical or radiologic diagnosis of recurrent disease in $90 \%$ of patients $(18,19)$.

Another marker, the beta-subunit of human chorionic gonadotropind, has also been reported to be a prognostic factor independentof stage and histology in patients with PFTC (20), but further studiesare needed.

\section{PATHOLOGICAL DIAGNOSIS}

The tubal carcinomas show characteristically fusiform swelling indistinguishable from hydrosalpinx or hematosalpinx. They mostly occurs in ampulla and occasionally, originate from the fimbrial end. Bilaterality is reported in $3 \%$ to $20 \%$ of cases. The disease is often advanced, but it is long confined to the peritoneal cavity (6,11,21).

Pathology remains the mainstay for diagnosis of PFTC. Serous carcinoma of the fallopian tube is the most common histological type of tubal carcinoma. It is an invasive tumour growing in papillary, glandular, and solid patterns with high grade nuclear atypia. These tumours are identical to their ovarian counterparts.

The second most common type of tumour is the endometrioid carcinoma, followed by undifferentiated, clear cell, mucinous, and transitional carcinomas.

PFTC is graded according to its differentiation and extent of solid components, with most tumours being poorly differentiated (9).

Initial diagnostic criteria for the diagnosis of PFTC were proposed by Hu et al. in 1950 (22). These were revised later by Sedlis et al. (23) and currently include the following: 1 . The main tumour arises from the endosalpinx; 2 . The histological pattern reproduces the epithelium of the tubal mucosa; 3 . The transition from benign to malignant tubal epithelium is demonstrable; 4. The ovaries or endometrium are either normal or contain a tumour that is smaller than the tumour in the tube. Recently, Singh et al. (24), proposed an approach to the pathological assignment of primary site in highgrade serous tubal, ovarian, and peritoneal carcinoma. According to Singh, the identification of tumour inside the fallopian tubes, even in the presence of larger tumours in other localizations, supports the diagnosis of PFTC (24).

The immunophenotype of adenocarcinoma of the fallopian tube is similar to that of ovarian carcinoma of the same histologic type. Primary tubal carcinomas are generally CK7 positive and CK20 negative. They typically display strong and diffuse nuclear staining for PAX-8 (25).

Before the development of molecular techniques, the classification of solid tumors was primarily based on site of origin and microscopic histological appearance. The development of immunohistochemical staining and fluorescence in situ hybridization (FISH) technology revolutionized the study of tumor biology, and both techniques have allowed for the sub-classification of tumors based on the presence of molecular biomarkers. Biomarker-based classification may provide prognostic information and may also influence the selection of adjuvant therapy (26).

\section{STAGING}

The currently accepted staging system for PFTC was developed by the International Federation of Gynecology and Obstetrics (FIGO), and currently PFTC, ovarian, and peritoneal cancers are staged collectively within the same system $(9,27)$.

Dissemination of PFTC occurs through the transcoelomic route with implantation of cells throughout the abdominal cavity, similarly to ovarian cancer, 
and also through continuity to adjacent organs, transluminal migration, haematogenous, and lymphatic spread. Distant metastases are more common in PFTC than ovarian cancer.

PFTC are more frequently detected in an earlier stage thandoesovarian cancer. $50 \%$ of patients with PFTC are diagnosed with stages I/ Ildisease (9).

Data from the literature indicate that patients with PFTC have a higher rate of retroperitoneal and distant metastases than those with EOC. PFTC is richly permeated with lymphatic channels that drain into the para-aortic lymph nodes through infundibulopelvic lymphatics, with involvementin $33 \%$ of patients with all stages, and almost equal involvement of the para-aortic and pelvic lymph nodes (9). Because lymph node metastases are common in patients with PFTC, lymphadenectomy is highly recommended for these patients. Kleinet al (17) found that radical lymphadenectomy increased the median survival to 43 months, compared to 21 months without lymphadenectomy, suggesting that radical lymphadenectomy in tumors of equal size may markedly prolong survival. Koo et al (28) also suggested that comprehensive retroperitoneal lymphadenectomy, including both para-aortic and pelvic lymphadenectomy, is an important procedure. Furthermore, Deffieux et al (29) described that the left para-aortic chain above the level of the inferior mesenteric artery was the site most frequently involved, even when the fallopian tubal cancer was onthe opposite side.

\section{TREATMENT}

Current management of PFTC follows the same guidelines as ovarian cancer in terms of surgical staging, debulking and adjuvant chemotherapy. The goal of the treatment is the complete removal of the tumor. The surgical approach consists of total abdominal hysterectomy, bilateral salpingo-oophorectomy, infra-colic omentectomy, appendicectomy, peritoneal washings, and peritoneal biopsies (2).

In addition to FIGO stage, complete resection with no or minimal residual tumors have also been reported to be the most important independent prognostic factor for both disease-free survival and overall survival (9). Klein et al (17) found that complete resection, including additional radical lymphadenectomy, provided a remarkably better 5-year survival rate of $83 \%$, whereas patients with total hysterectomy and bilateral salpingo-oophorectomy achieved a 5-year survival rate of only $58 \%$.

Surgery without lymphadenectomy may result in underestimating the clinical stage and metastases of the lymph nodes must be expected in $30 \%$ of cases intraoperatively considered as stage II $(9,30)$.

The high rate of lymph node metastases makes PFTC appear to be a generalized systemic disease even in early-stage PFTC, and this justifies the intensified use of chemotherapy. However, some authors considered that stages IA and IB disease may not require extra adjuvant treatment, except early-stage patients with tumors infiltrating the serosa or with preoperativelyor intraoperatively ruptured tumors (12). The combination of platinum and a taxane has been a cornerstone of treatment of PFTC for more than 15 years. The addition of a third cytotoxic drug, whether in triplet combinations or sequential doublets, provides no benefit. However, improvement might come through changes in scheduling, dose intensity ordelivery. Several authors using cisplatin-based chemotherapy for patients with advanced-stage PFTC reported overall response rates of $53-92 \%(31,32)$.

The response rate of cisplatin-based chemotherapy is 53 to $92 \%$. There are few data on the response to this chemotherapy in advanced stages (11).

Pectasides et al. (12), in a series of 64 patients, obtained an overall response rate of $93 \%$ (best in all publications) for a combination of platinum-based chemotherapy with carboplatin and paclitaxel. Peters et al. reported a complete response rate of $75 \%$ in a study of 46 patients. Similarly, in a series of 45 patients treated with cisplatin, Gadducci et al. (1), reported rates of complete and partial response of $64.4 \%$ and $17.8 \%$ respectively, with a five-year survival of $56 \%$ for complete response and only 21 months for partial response.

Hormonal therapies may be of value in the future, given the sensitivity and response of the fallopian tube epithelium to hormonal fluctuations, although there are no current recommendations (9).

\section{OUTCOMES}

The 5-year survival rate of PFTC ranges between $22 \%$ and $57 \%(9,33,34)$.

The traditional belief is that the survival rates of patients with PFTC are poor, and worse than those of patients with equivalent stages of serous epithelial ovarian carcinoma (SEOC) or other early-stage gynecologic malignancies. However, the lack of complete staging surgery among early-stage FIGO Stage I/II patients may have underestimated FIGO staging (35).

The National Cancer Institute's Surveillance Epidemiology and End Results program reported that $47 \%$ of patients identified as having Stage I/II PFCT had neither a para-aortic nor a pelvic lymph node evaluation 
(2). In addition, Moore KN et al (36) demonstrated better outcomes of early stage PFTC than those of SEOC, for the 5 -year overall survival (95\% vs. $76 \%$ ) and also 5year progression-free survival rates ( $79 \%$ vs. $65 \%$ ).

The prognostic factors identified for increased survival include stage, age, and residual tumour after surgery, serous subtype and elevated pretreatment CA-125 (1,9).

Many authors have suggested that the FIGO stage is the most important independent prognostic factor for patients with PFTC. Kosary and Trimble (2) found that 5 -year survival by FIGO stage was $95 \%$ in stage I, $75 \%$ in stage II, $69 \%$ in stage III and $45 \%$ in stage IV. Wethington et al (37) also reported similar findings: the 5-year survival rate for Stage I tumors was $81 \%$, and cancerspecific survival was $65 \%$ and $54 \%$ for Stages II and III, respectively.

Recurrent disease, which occurs on average 2 to 3 years after initial treatment, is associated with a poor prognosis due to the lack of alternative treatments available $(6,9)$.

Although PFTC is very similar to serous-type EOC, there are still a few differences between the two. Abdominal pain is often found in patients with PFTC, because of the tubal distension (6). The shorter history of symptoms in PFTC than in EOC allows detection at an earlier stage in patients with PFTC than in EOC. PFTC shows a propensity for microscopic distant metastases, compared with the macroscopic intraperitoneal metastasis of EOC and PFTC tends to recur in the retroperitoneal nodes and distant sites more than does EOC $(35,38,39)$.

Similar to EOC (40), complete surgical staging in early-stage (FIGO I/II) PFTC and extensive and optimal debulking surgery in late stage (FIGO III/IV) PFTC, including cytology, total hysterectomy, bilateral salpingooophorectomy, retroperitoneal lymph node dissection, appendectomy, omentectomy and excisional biopsy for all suspicious lesions, provides the best chance of cure. Because of the propensity of PFTC for microscopic distant metastases and the relatively high risk of recurrence despite complete tumor excision in the early stage, postoperative chemotherapy is highly recommended $(7,34)$.

\section{CONCLUSIONS}

Primary fallopian tube cancer is a rare and challenging entity for both clinician and pathologist as similar morphology can be seen in both uterine and ovarian primaries with secondary extension to the fallopian tube. PTFC is often an earlier FIGO stage than is EOC, because of the appearance of earlier but non- specific symptoms or signs. The therapeutic strategy, which is often based on the guidelines for treatment of EOC, includes complete surgical resection or optimal cytoreductive surgery with minimal residual tumor, followed by platinum-paclitaxel combination chemotherapy with/without targeted therapy.

Targeted therapies will likely be a significant component of future treatment regimens for gynecologic malignancies. The commercial use of next-generation sequencing to generate personalized profiles of patient tumor samples has entered clinical practice and is being used to guide treatment decisions.

\section{REFERENCES}

1. Gadducci A, Landoni F, Sartori E, Maggino T, Zola P, Gabriele A et al. Analysis of treatment failures and survival of patients with fallopian tube carcinoma: a cooperation task force (CTF) study. Gynecol Oncol. 2001 May;81(2):150-159.

2. KosaryC,Trimble EL. Treatment and survival for women with Fallopian tube carcinoma: a population-based study. Gynecol Oncol. 2002 Aug;86(2):190-191.

3. Baekelandt M, Jorunn NA, Kristensen GB, Trope CG, Abeler VM. Carcinoma of the fallopian tube. Cancer. 2000 Nov 15;89(10):20762084.

4. Nappi R, Resta L, Nappi L, Loizzi P. Primary carcinoma of the fallopian tube: report on two cases. Eur J Obstet Gynecol Reprod Biol. 1996 Dec;70(1):93-96.

5. Deffieux X, Morice P, Thoury A, Camatte S, Duvillard P, Castaigne D. Pelvic and para-aortic lymphatic involvement in tubal carcinoma: topography and surgical implications. Gynecol Obstet Fertil. 2005 Jan; 33(1-2):23-28.

6. Kalampokas E, Kalampokas T, Tourountous I. Primary fallopian tube carcinoma. Eur J Obstet Gynecol Reprod Biol. 2013 Jul;169(2):155161.

7. Wang PH, Yuan CC, Chao HT. Surgico-pathological stage I primary fallopian tube adenocarcinoma. Int J Gynaecol Obstet. 1998 Oct; 63(1):73-74.

8. Pusiol T, Piscioli F, Morelli L, Parolari AM, Licci S. Cervicovaginal smears in the diagnosis of asymptomatic primary fallopian tube carcinoma. Cytopathology. 2009 Dec;20(6):409-411.

9. Lopes DJ, Cunha TM, Gomes FV, Calle C, Felix A. Neuroendocrine tumours of the female genital tract: a case-based imaging review with pathological correlation. Insights Imaging. 2015 Feb;6(1):43-52.

10. Ma FH, Cai SQ, Qiang JW, Zhao SH, Zhang GF, Rao YM. MRI for differentiating primary fallopian tube carcinoma from epithelial ovarian cancer. J Magn Reson Imaging. 2015 Jul;42(1):42-47.

11. BoufettalH,Samouh N. Primary fallopian tube carcinoma: a case report. Pan Afr Med J. 2014;18:263-

12. Pectasides $D$, Pectasides $E$, Economopoulos $T$. Fallopian tube carcinoma: a review. Oncologist. 2006 Sep;11(8):902-912.

13. Spencer JA, Forstner R, Cunha TM, Kinkel K. ESUR guidelines for MR imaging of the sonographically indeterminate adnexal mass: an algorithmic approach. Eur Radiol. 2010 Jan;20(1):25-35.

14. Kim MY, Rha SE, Oh SN, Jung SE, Lee YJ, Kim YS et al. MR Imaging findings of hydrosalpinx: a comprehensive review. Radiographics. 2009 Mar;29(2):495-507.

15. Ghattamaneni S, Bhuskute NM, Weston MJ, Spencer JA. Discriminative MRI features of fallopian tube masses. Clin Radiol. 2009 Aug;64(8):815-831.

16. RezvaniM,Shaaban AM. Fallopian tube disease in the nonpregnant patient. Radiographics. 2011 Mar;31(2):527-548.

17. Klein M, Rosen AC, Lahousen M, Graf AH, Rainer A. Lymphadenectomy in primary carcinoma of the Fallopian tube. Cancer Lett. 1999 Dec 1;147(1-2):63-66

18. Hefler LA, Rosen AC, Graf AH, Lahousen M, Klein M, Leodolter S et al. 
The clinical value of serum concentrations of cancer antigen 125 in patients with primary fallopian tube carcinoma: a multicenter study. Cancer. 2000 Oct 1;89(7):1555-1560.

19. Shamshirsaz AA, Buekers T, Degeest K, Bender D, Zamba G, Goodheart MJ. A single-institution evaluation of factors important in fallopian tube carcinoma recurrence and survival. Int J Gynecol Cancer. 2011 Oct;21(7):1232-1240.

20. Riska A, Alfthan H, Finne P, Jalkanen J, Sorvari T, Stenman UH et al. Preoperative serum hCGbeta as a prognostic marker in primary fallopian tube carcinoma. Tumour Biol. 2006;27(1):43-49.

21. Hosokawa C, Tsubakimoto M, Inoue Y, Nakamura T. Bilateral primary fallopian tube carcinoma: findings on sequential MRI. AJR Am J Roentgenol. 2006 Apr;186(4):1046-1050.

22. Hu CY, Taymor ML, Hertig AT. Primary carcinoma of the fallopian tube. Am J Obstet Gynecol. 1950 Jan;59(1):58-67, illust.

23. Sedlis A. Carcinoma of the fallopian tube. Surg Clin North Am. 1978 Feb;58(1):121-129.

24. Singh N, Gilks CB, Wilkinson N, McCluggage WG. Assignment of primary site in high-grade serous tubal, ovarian and peritoneal carcinoma: a proposal. Histopathology. 2014 Aug;65(2):149-154.

25. Hashi A, Yuminamochi T, Murata S, Iwamoto H, Honda T, Hoshi K. Wilms tumor gene immunoreactivity in primary serous carcinomas of the fallopian tube, ovary, endometrium, and peritoneum. Int J Gynecol Pathol. 2003 0ct;22(4):374-377.

26. Hillman RT, Ward K, Saenz C, McHale M, Plaxe S. Barriers prevent patient access to personalized therapies identified by molecular tumor profiling of gynecologic malignancies. J Pers Med. 2015;5(2):165173.

27. Prat J. Staging classification for cancer of the ovary, fallopian tube and peritoneum. Int J Gynaecol Obstet. 2014 Jan;124(1):1-5.

28. Koo YJ, Kwon YS, Lim KT, Lee KH, Shim JU, Mok JE. Para-aortic lymphadenectomy for primary fallopian tube cancer. Int J Gynaecol Obstet. 2011 Jan;112(1):18-20.

29. Deffieux X, Morice P, Thoury A, Camatte S, Duvillard P, Castaigne D. Anatomy of pelvic and para-aortic nodal spread in patients with primary fallopian tube carcinoma. J Am Coll Surg. 2005 Jan; 200(1): 45-48.

30. Caldeira JP, Cunha TM. Fallopian tube carcinoma. Eurorad - European Radiology Online Database-BCertified Radiological Teaching Cases. 2008.
31. Katsumata N, Yasuda M, Isonishi S, Takahashi F, Michimae H, Kimura $\mathrm{E}$ et al. Long-term results of dose-dense paclitaxel and carboplatin versus conventional paclitaxel and carboplatin for treatment of advanced epithelial ovarian, fallopian tube, or primary peritoneal cancer (JGOG 3016): a randomised, controlled, open-label trial. Lancet Oncol. 2013 Sep;14(10):1020-1026.

32. Bookman MA, Brady MF, McGuire WP, Harper PG, Alberts DS, Friedlander $\mathrm{M}$ et al. Evaluation of new platinum-based treatment regimens in advanced-stage ovarian cancer: a Phase III Trial of the Gynecologic Cancer Intergroup. J Clin Oncol. 2009 Mar 20;27(9): 1419-1425.

33. Horng HC, Teng SW, Lai CR, Chang WH, Chang YH, Yen MS et al. Prognostic factors of primary fallopian tube cancer in a single institute in Taiwan. Int J Gynaecol Obstet. 2014 0ct;127(1):77-81.

34. Rosen AC, Reiner A, Klein M, Lahousen M, Graf AH, Vavra $\mathrm{N}$ et al. Prognostic factors in primary fallopian tube carcinoma. Austrian Cooperative Study Group for Fallopian Tube Carcinoma. Gynecol Oncol. 1994 Jun;53(3):307-313.

35. Horng HC, Lai CR, Chang WH, Wen KC, Chen YJ, Juang CM et al. Comparison of early-stage primary serous fallopian tube carcinomas and equivalent stage serous epithelial ovarian carcinomas. Taiwan $\mathrm{J}$ Obstet Gynecol. 2014 Dec;53(4):547-551.

36. Moore KN, Moxley KM, Fader AN, Axtell AE, Rocconi RP, Abaid LN et al. Serous fallopian tube carcinoma: a retrospective, multi-institutional case-control comparison to serous adenocarcinoma of the ovary. Gynecol Oncol. 2007 Dec;107(3):398-403.

37. Wethington SL, Herzog TJ, Seshan VE, Bansal N, Schiff PB, Burke WM et al. Improved survival for fallopian tube cancer: a comparison of clinical characteristics and outcome for primary fallopian tube and ovarian cancer. Cancer. 2008 Dec 15;113(12):3298-3306.

38. Pectasides D, Pectasides E, Papaxoinis G, Andreadis C, Papatsibas G, Fountzilas $\mathrm{G}$ et al. Primary fallopian tube carcinoma: results of a retrospective analysis of 64 patients. Gynecol Oncol. 2009 0ct;115(1):97101.

39. Ajithkumar TV, Minimole AL, John MM, Ashokkumar OS. Primary fallopian tube carcinoma. Obstet Gynecol Surv. 2005 Apr;60(4):247-52.

40. Chao KC, Chen YJ, Juang CM, Lau HY, Wen KC, Sung PL et al. Prognosis for advanced-stage primary peritoneal serous papillary carcinoma and serous ovarian cancer in Taiwan. Taiwan $\mathrm{J}$ Obstet Gynecol. 2013 Mar;52(1):81-84. 